\title{
Effect of Reading and Writing Based Treatment Approaches on Verbal Output
}

\author{
Pinki Sing ${ }^{1}$ and Apoorva Pauranik ${ }^{2}$ \\ ${ }^{1}$ Department of ENT, All India Institute of Medical Sciences (AIIMS), Bhopal, India \\ ${ }^{2}$ Department of Medicine, MGM College and Hospital, Indore (M.P), India
}

Corresponding author: Pinki Singh, Ph. D Scholar, MASLP, Speech Language Pathologist, Department of ENT, All India Institute of Medical Sciences, Bhopal, Pin 462024, India, Tel: 09302751995; E-mail: pinkiaslp53@gmail.com

Received: April 21, 2017; Accepted: May 22, 2017; Published: May 29, 2017

Citation: Singh P, Pauranik A. Effect of Reading and Writing Based Treatment Approaches on Verbal Output. J Neurol Neurosci. 2017, 8:3.

\section{Abstract}

Background: Impairments in speech-language and communication in person with aphasia (PWA) can be parsed into various sub-modules spanning across hierarchy of different linguistic and its input-output modalities. The modules are partially interdependent and overlapping, some more or less. Impairment based therapies have varying degree of generalization effect across the modality and hierarchy. Literate PWA has higher potential for recovery with or without therapy; which might be due to cross model transfer of competence.

Aim: To understand the implication of simultaneous usage of reading-writing approaches and its generalization across to the modality of verbal.

Methods: case study design, treatment was initiated on reading and writing tasks from word to simple sentence level, whereas generalization was tested in picture confrontation naming for nouns, verbs and spontaneous speech during picture description and narrative task.

Results and discussion: Performance on functional communication, aphasic quotient and mean length utterances improved significantly along with reading and writing skills. This exemplifies the fact that therapeutic practices in reading and writing domains may have significant beneficial effect. Even results from several nonidentical patients can be accounted for by the same assumption, which could be reinforced rather than weakened by the diversity of results.

Conclusion: In conclusion, findings from this exploratory study indicate that the simultaneous use of reading and writing approaches is useful in improving verbal skills. To understand the potential of this approach, it should be explored and documented through single -case studies.

Keywords: Aphasia; Reading; Writing; Theory

\section{Introduction}

Aphasia, a language disorder following acquired brain damage, most frequently occurs after a stroke in the dominant language hemisphere of the brain. Between 21 and 38 percent of all stroke survivors exhibit aphasia [1]. Impairments in speech-language and communication in person with aphasia (PWA) can be parsed into various sub-modules spanning across the hierarchy of different linguistic and its input-output modalities. The modules are partially interdependent and overlapping, some more or some less. Impairment-based therapies are aimed at improving language functions and consist of procedures in which the clinician directly stimulates specific listening, speaking, reading and writing skills. Communication-based (also called consequence-based) therapies are intended to enhance communication by any means and encourage support from caregivers. These therapies often consist of more natural interactions involving real life communicative challenges. Impairment-based therapies are known to have varying degrees of generalization effects across the modality and hierarchy. Literate and better educated PWA has a higher potential for recovery with or without therapy; which might be due to cross model transfer of competence. It is debatable as to whether we should concentrate on impairment or communication-based therapies should try to tackle the questions related to cross model transfer of competence and its role in improvement through simultaneous usage of reading-writing approaches and its generalization across the modality.

To define the word "theory" is not easy. A theory comprises the general principles of science, a system of ideas explaining something; it should account for the existing data and predict outcomes that have not yet been tested. The first and most important step in the construction of a theory of rehabilitation is to agree on some basic principles and accumulate knowledge as it becomes available. A cooperative effort is necessary for building a common framework to which to add all new knowledge coming from different sources and disciplines.

As we know, aphasia therapy has not progressed sufficiently to support the development of a fully articulated theory of aphasia rehabilitation. Mitchum et al. [2], stated that "In our view, it is premature to attempt to propose a theory of 
therapy, especially in light of the limited detail presented in the models of language processing that are available". However, merely collecting data and refining models of language processing will not lead to the foundation of a theory.

Caramazza and Hillis [3] discussed the framework for a remediation theory and argued that the development of such a theory requires a model of the cognitive system to be treated, a detailed hypothesis about the damage in the patients to be treated, and a motivated hypothesis about how therapy modifies the damaged processes.

In an effort to understand representation and processing of language, neuroscience research has demonstrated that when networks of neurons in the brain are frequently simultaneously active, they become strongly connected with each other. In contrast, asynchronous firing weakness established synaptic links [4,5]. The correlation learning implied by the "fire together wire together rule" has implications for the representation and processing of language in the human brain [6]. Conversely, if connected neurons are frequently active independently from each other, their connections undergo synaptic weakening. Such "anti-Hebb" learning [5] may become relevant to language in cases of brain injury. For instance, sometimes partial damage to the circuit connecting word form \& concept does not allow the conceptual circuit to spark the linguistic one, and vice versa. The use of an inappropriate word may lead to further antiHebb learning, which could, therefore, imply the further weakening of the already damaged word-concept links $[7,8]$.

These considerations have clear implications for neurorehabilitation. As much as co-activation of crucial neuronal circuits should be encouraged, the independent activation of circuits' parts could be avoided.

\section{Need for the Study}

There are several studies which have been devoted to these questions e.g. how are recovery from brain injury and recovery from language disorders related? Does aphasia therapy affect brain changes? The results so far have only been descriptive and often contradictory. Even, neural mechanisms underlying recovery from aphasia as well as from other cognitive disorders are still an open question. The human central nervous system has only limited potential for regeneration and in recovery from aphasia, the relative contributions of behavioural adaptation and of true neurological recovery are unclear. An important question is whether we should concentrate on the most prominent kind of aphasia therapy or should try to tackle the question of the underlying causes and mechanism of improvement to facilitate language and communication of PWA.

\section{Aim of the Study}

To understand the implication of the simultaneous use of reading and writing approaches and its generalization effects across the verbal modality.

\section{Methods}

\section{Participants}

Two participants; 55 years/male with moderate anomic aphasia post stroke 13 years and 22 years/female with severe Broca's aphasia post traumatic brain injury with acquired dyslexia and agraphia participated.

\section{Materials}

Naming, repetition, advanced discourse level, advanced reading and advanced writing sections of Manual for Adult Aphasia (MANAT).

\section{Procedure}

A nominal picture was shown to the participant and asked to name it; if successful, (s) he was simply asked to repeat the name once and then to write down the name since some patients get succeeded in writing the first letter(s) of a word they have been unable to name. Generally, however, patients cannot write any of the letters. The therapist then wrote the first letter and patient was asked to complete the word without speaking. If successful, the patient was asked to read the word and then the whole procedure was repeated, that is, the patient was asked again to name the picture and then write it down (without being allowed to see the previously written word). When, as is often the case, the patient didn't complete the word after the therapist has written the first letter, the therapist wrote the second letter and once more asked the patient to complete it without saying the word. The same procedure is followed until the patient completed the written word or, in the case of total failure, copies the entire word written by the therapist. Immediately after writing the word, the patient was asked to say it and then to write it without being allowed to copy it.

When the patient produced a correct written response, then he or she could read it through the (undamaged) graphemeto-phoneme conversion mechanism. If the word has a regular orthography, the output of the conversion mechanisms corresponds exactly to the phonological representation of the word in the phonological output lexicon.

The orthographic cue has proven to be effective in facilitating naming by patients [9-11], but it was not the only effective one. Participants were also suggested to work at home with a small dictionary, as for the input lexicons, however, the first few sessions were carried out with the help of the therapist in order to teach the patient how to perform the task. The patient selected a letter and described all the words, then they said the words again and wrote down (at this stage, misspellings were corrected by the therapist). When the participant could not think of any more words, he or she rewrote them in alphabetical order and then looked up in the dictionary the first words started with the letter chosen. As for rehabilitation of the input lexicons, the participant disregarded the words that do not seem familiar and searched only for the 
familiar ones. The participant read the dictionary definition of the chosen word and, if that word seemed important, he or she wrote it down with the other ones. In this way, the patient added a limited number of words per day. On the following day, the first step is to try to remember all the words practiced the day before and write them down. The participant then checked whether any words have been forgotten or not? Any forgotten words would be added to the newly written list, and the participant has to practice it again. When this is done, with the help of the dictionary the patient added a few words. The same was done each day. Then, to facilitate fluency simultaneously, picture tasks were introduced having nominal and verbs, which have been practiced before to make a spontaneous speech during picture description and narrative task easier.

\section{Mechanism behind the logic of usage of reading and writing task prior to focus on fluency and narrative skills}

The mechanisms involved in phonological and orthographic processing are computationally independent. In reading, for instances, visual information must be converted into orthographic information, which, in turn, must be processed lexically; in comprehending oral language, lexical information must be extracted from the phonemic input, which is processed from acoustic input.

In production, initial information is semantic for both oral and written output. In oral production, it must then be transformed into a phonological abstract representation of the word that must finally be articulated, whereas in written production it may be transformed into an abstract orthographic representation and then into graphic movements.

Even though not much research has been carried out on the mechanisms of written word production, and it has long been maintained that orthographic knowledge is fundamentally parasitic upon phonological knowledge. The phonological mediation hypothesis states that in reading for comprehension, it is necessary to generate the internal phonological representation of the word's written form before accessing its meaning. In spelling, it is argued that the orthographic form of the word cannot be directly accessed from the semantic system; access to the orthographic representation is gained through the phonological lexicon.

Goodglass and Hunter [12] compared oral and written production in two patients, one with Broca aphasia and the other with Wernicke aphasia. They found that the oral and written productions of each patient were similar but they differed between the two patients. The qualitative similarity between the oral and written productions in either of the two patients and the opposite patterns they showed of the patients were considered evidence that "written language is, at least in part, the formulation of spoken language converted to graphic form". However, a few patients have been described with better -preserved written naming than oral naming. Lhermitte and Derousesne [13] have described the two cases of this kind. One patient with left-hemisphere vascular lesions produced many phonemic errors in oral production, whereas her writing was correct. The Second patient, who had head trauma, correctly named only 3 of 50 pictures in the spoken modality; most of his errors were neologisms. In the written modality he correctly named 44 of the same 50 pictures. For bicyclette (bicycle), for instance, he said "fogran" and wrote (bicyclette); for peigne (comb) he said "bradin" and wrote (peigne). It is interesting to note that the authors did not consider that their results demonstrated the independence of the orthographic representations from phonological mediation. On the contrary, they argued that it was not possible to write without previously formulating orally what one wants to express. To explain the observed dissociation between better written than oral naming and production in these patients, they hypothesized a disconnection between (unimpaired) abstract language capacity and its (unimpaired) articulatory implementation.

To sum up, it could be assumed that there are three main distinctions in lexical knowledge types: semantic, phonological, and orthographic. In addition, it is assumed that phonological and orthographic knowledge is modality specific and that the lexical subcomponents are interrelated through a single semantic system.

\section{Result and Discussion}

In Table 1, participant's profiles are mentioned. The data derived from the treatment probes during baseline and treatment phases of the study are illustrated in Table 2 . Results of each task will be discussed separately.

Table 1 Participant's profile.

\begin{tabular}{|l|l|l|}
\hline \multirow{2}{*}{ Characteristics } & Participant 1 & Participant 2 \\
\cline { 2 - 3 } & G K G & J B \\
\hline Sex & M & F \\
\hline Age & 64 & 20 \\
\hline Education (years) & 16 & 13 \\
\hline $\begin{array}{l}\text { Handedness (pre/post } \\
\text { stroke) }\end{array}$ & R/R & R/R \\
\hline Time since onset (years) & 13 & 1.3 \\
\hline Aphasia type & Anomic aphasia & Broca's aphasia \\
\hline Physical limitation & No & No \\
\hline
\end{tabular}


Table 2 Participant performance on language domains specific task: Pre and Post-treatment.

\begin{tabular}{|c|c|c|c|c|}
\hline \multirow[t]{3}{*}{ Task } & \multicolumn{2}{|l|}{ Participant 1} & \multicolumn{2}{|l|}{ Participant 2} \\
\hline & \multicolumn{2}{|l|}{ G K G } & \multicolumn{2}{|l|}{ J B } \\
\hline & Pre-treatment & Post-treatment & Pre- treatment & Post- treatment \\
\hline Naming & $40 / 106$ & $85 / 106$ & $15 / 106$ & $81 / 106$ \\
\hline Repetition & $20 / 20$ & & $10 / 20$ & $17 / 20$ \\
\hline Advanced discourse level & IC- 5 , FGP- 4 & IC- 8, FGP- 9 & IC- 3 , FGP- 3 & IC-9, FGP- 9 \\
\hline \multicolumn{5}{|l|}{ Advanced Reading } \\
\hline Recognition of all alphabets at word level & $30 / 30$ & - & $30 / 30$ & - \\
\hline Reading at the level of phrase and Sentence & Good & - & Fair & Good \\
\hline Reading Comprehension & NR & $\begin{array}{l}\text { (Level.1\&2): } \\
\text { 100\% } \\
\text { (Level. 3): } \\
\text { Task I-7/9; } \\
\text { Task II-5/5) }\end{array}$ & $\begin{array}{l}\text { Level. 1: } \\
3 / 6 \\
1 / 4 \\
\text { Levels } 2 \text { and 3: NR }\end{array}$ & $100 \%$ \\
\hline \multicolumn{5}{|l|}{ Advanced Writing } \\
\hline Written Naming & $1 / 10$ & $5 / 10$ & $0 / 10$ & $10 / 10$ \\
\hline Writing to Dictation & $\begin{array}{l}\text { 3/10 (simple words), } \\
0 / 10 \text { (complex words) }\end{array}$ & $7 / 10,4 / 10$ & $0 / 10$ & $7 / 10$ \\
\hline Sequence/Procedural task & NR & Fair & NR & Content Good \\
\hline
\end{tabular}

\section{Advanced reading}

As seen in Table 2, during baseline, no response was found for GKG on reading comprehension tasks. Following treatment, reading comprehension ability gradually improved to $100 \%$ correct for level 1 and 2 tasks. For JB, baseline responses on reading task at the level of phrase and sentence were fair along with 'fair response' on level 1 and 'no response' on level 2 and 3 reading comprehension tasks. Following treatment, JB improved from 'fair' to 'good' on reading ability of phrase and sentences. JB baseline scores of reading comprehension also improved to $100 \%$ accuracy.

\section{Advanced writing}

As seen in Table 2, written naming of stimuli during baseline was 0 to $1 \%$ for both the participants. Following treatment, generalization to these items was observed with written naming improving to $50 \%$ and $100 \%$ accuracy for GKG and JB respectively. On writing to dictation task, JB has improved markedly for simple and complex words from baseline to final treatment phase whereas GKG had a fair response. Both participants had given no response on sequence/procedural task during baseline assessment, which got improved to fair and good content for GKG and JB respectively [14].

\section{Naming, repetition and advanced discourse level}

Additionally, for GKG generalization to the untrained tasks e.g. Naming and Advanced discourse were noted from 40/106 during baseline to $85 / 106$ at the final treatment phase; Information content (IC)-5, Fluency, Grammatical Competence, and Paraphasias (FGP)-4 during baseline to IC-8, FGP-9 at the final treatment phase respectively were found. For JB generalization to the Naming, Repetition and Advanced discourse were also noted from $15 / 106$ to $81 / 106,10 / 20$ to $17 / 20$ and IC-3, FGP-3 to IC-9, FGP-9 respectively for the baseline to the final treatment phase.

Cognitive neuropsychologist stressed the rigorous study of single patients and abandoned the group study approach; they argued for a functional approach to the study approach; they argued for a functional approach to the study of the mind explicitly independent of the study of the brain, and they introduced the use of information -processing models, which provide a rational basis for the characterization of pattern of impaired performance in terms of damaged sub-components.

If the cognitive system were not very similar (if not exactly the same) in every one of us, data from different patients would point to two or more different cognitive structures. If, on the contrary, as has happened, results from the various persons with aphasia suggest the same cognitive structure, the assumption of universality is reinforced. The degree to which patients performances converge and are interpretable by 
making similar assumptions is an indication of the extent to which the proposed theory of the normal cognitive structure is confirmed by patient's data.

In most cases, patients get rehabilitated through impairment based therapies or communication- based therapy e.g. anomia has been required to produce the target words but the strategies used would have differed. The techniques most frequently used by therapists are phonemic or semantic cueing (more rarely an orthographic cue), repetition, reading, and word-picture matching $[15,16]$. Among the facilitation techniques used, the phonemic cue (saying the first phoneme or syllable of a to-be-named word) has been generally found to be the most efficacious, but its facilitating effect is short-lived [17].

\section{Conclusion}

In conclusion, findings from this exploratory study indicate that simultaneous use of reading and writing approaches is useful in improving verbal skills. Even though we do not have a definite approach to facilitate generalization effect on verbal modality for different type and severity of aphasia, the present treatment concept may be applicable for all type of person with aphasia (PWA). To understand the potential of this approach, it should be explored and documented through single -case studies.

\section{Criticism and Assumption}

The average results of two or more patients are not feasible because the functional lesions are different and interfere differently with the execution of the experimental task [18]. Thus, it is impossible to replicate single- cases studies since no two brain lesions are exactly the same and it is impossible to find two patients who show exactly the same functional damages. But this is not an insurmountable obstacle because Replicability can be done within the patient if crucial findings can be replicated within the same patient on more than one occasion. Replicability across patient is made possible by the use of the multiple single cases studies method. When results from several non-identical patients can be accounted for by the same theory about the normal cognitive mechanism, the theory could be reinforced rather than weakened by the diversity of results.

\section{Limitation}

This approach would be only effective for a literate subject with non-fluent aphasia and not for a subject with fluent aphasia where comprehension has got compromised. Its outcome would be dependent on personal factors, injuryrelated factors, cognitive factors, learning (errorless learning was superior to trial-and-error learning) too.

\section{References}

1. Laska AC, Hellbom A, Murray V, Kahan T, Von Arbin M (2001) Aphasia in acute stroke and relation to outcome. J Intern Med 24: 413-422.

2. Mitchum CC, Greenwald ML, Berndt RS (2000) Cognitive treatments of sentence processing disorders: What have we learned? Neuropsychol Rehabil 10: 311-336.

3. Caramazza A, Hillis AE (1993) For a theory of remediation of cognitive deficits. Neuropsychol Rehabil 3: 217-237.

4. Hebb DO (1949) The organization of behavior. A neuropsychological theory. John Wiley \& Sons, New York.

5. Tsumoto $T$ (1992) Long term potentiation and long term depression in the neocortex. Progress in Neurobiology 39: 209-228.

6. Pulvermuller $F$ (1999) Words in the brain's language. Behav Brain Sci 22: 253-336.

7. Berthier ML, Pulvermuller F (2011) Neuroscience insights improve neurorehabilitation of post stroke aphasia. Nat Rev Neuro 7: 86-97.

8. Pulvermuller F, Berthier ML (2008) Aphasia therapy on a neuroscience basis. Aphasiology 22: 563-599.

9. Best WM, Hickin J, Herber R, Howard D, Osborne F (2000) Phonological facilitation of aphasia naming and predicting the outcome of treatment for anomia. Brain Lang 74: 435-438.

10. Henaff Gonon MA, Bruckert R, Michel F (1989) Lexicalization in an anomic patient. Neuropsychologia 27: 391-407.

11. Bachy-Langedock N, De Partz MP (1989) Coordination of two reorganization therapies in a deep dyslexic patient with oral naming disorder. In: Seron X, Deloche G, (eds). Cognitive approaches in neuropsychological rehabilitation. Hillsdale, Lawrence Erlbaum Associates, New Jersey pp: 211-247.

12. Goodglass H, Hunter M (1970) A linguistic comparison of speech and writing in two types of apahsia. J Commun Disord 3: 28-35.

13. Lhermitte F, Derousesne J (1974) Paraphasies et jargonaphasic dans le language oral avec conservation du language ecrit. Revue Neurologique 130: 21-38.

14. Deshpande R, Goswami SP (2008) Manual for adult non-fluent aphasia therapy-in Hindi (MANAT-Hindi). Student Research Vol. II AlISH. A Publication of AlISH, Mysore.

15. Kremin H (1993) Is there more than ah-oh-ah? Alternative strategies for writing and repeating lexically. In: Coltheart $M$, Sartori G, Job R (eds). Foundations of aphasia rehabilitation. Oxford: Pergamon Press pp: 261-292.

16. Nickel L, Best W (1996) Therapy for naming disorders (Part I): Principles, puzzles and progress. Aphasiology 10: 21-47.

17. Patterson KE, Purell C, Morton J (1983) Facilitation of word retrieval in aphasia. In: Code C, Muller D, (eds). Aphasia therapy. London: Edward Arnold pp: 76-87.

18. Caramazza A, Mccloskey M (1988) The case for single-patient studies. Cogn Neuropsy 5: 517-528.

\section{Competing and Conflicting Interest}

No competing and conflicting interests exist. 